\title{
A LIMIT-POINT CRITERION FOR NONOSCILLATORY STURM-LIOUVILLE DIFFERENTIAL OPERATORS ${ }^{1}$
}

\section{HERBERT KURSS}

The main point of the present paper is to derive a limit-point criterion from which the criteria of Weyl [6], Friedrichs [3] and Sears [4] follow as special cases. This limit-point criterion is an immediate consequence of a Sturm comparison theorem. It is shown to be equivalent to the criterion of Weyl [6] when use is made of a Liouville-type transformation formula.

Consider the Sturm-Liouville differential operator

$$
L y=-\left(p y^{\prime}\right)^{\prime}+q y, \quad X^{-}<x<X^{+},
$$

where, in the open interval $\left(X^{-}, X^{+}\right), p(x)$ and $q(x)$ are real and continuous and $p(x)>0$.

Let $x_{0}$ be any point where

$$
X^{-}<x_{0}<X^{+}
$$

Defjnition (WeyL [6]). $L$ is of limit-point type (abbreviated LP) at $X^{+}$if for some constant $\lambda_{0}$ not all solutions of $L y=\lambda_{0} y$ are in $L^{2}\left(x_{0}, X^{+}\right)$.

A corresponding definition applies for $L$ being LP at $X^{-}$.

We will freely use the fact, proved by Weyl [6, p. 27], [7, p. 415] (see also [2]), that if all solutions of $L y=\lambda y$ are in $L^{2}$ for some $\lambda=\lambda_{0}$, then this is also the case for every $\lambda$ in the complex plane.

ThEOREM. $L$ defined by

$$
L y=-\left(p y^{\prime}\right)^{\prime}+q y
$$

is LP at $X^{+}$if there is a comparison operator

$$
\hat{L} v=-\left(p v^{\prime}\right)^{\prime}+\hat{q} v
$$

such that (i) $\hat{L}$ is LP and nonoscillatory at $X^{+}$and

(ii) $q-\hat{q}$ is bounded below at $X^{+}$.

Proof. By hypothesis (i) there is a solution, $v$, of $\hat{L} v=0$ which is strictly positive and not in $L^{2}$ on some interval $\left(x_{0}, X^{+}\right)$.

By hypothesis (ii) there is a constant $\lambda_{0}$ such that for all $x \geqq x_{0}$,

$$
q-\hat{q} \geqq \lambda_{0} \text {. }
$$

Presented to the Society, September 1, 1966; received by the editors June 24, 1966.

1 This research was completed under U. S. Navy Contract No. Nonr 3360 (01). 
Define $y$ to be the solution of $L y=\lambda_{0} y$ which satisfies the initial conditions

$$
y\left(x_{0}\right)=v\left(x_{0}\right), \quad y^{\prime}\left(x_{0}\right)=v^{\prime}\left(x_{0}\right) .
$$

Then by a Sturm comparison theorem (see, for example, [1] or [5])

$$
y(x) \geqq v(x) \quad \text { for } x \geqq x_{0} .
$$

Hence $y(x)$ is not in $L^{2}\left(x_{0}, X^{+}\right)$. Q.E.D.

It is easily seen that the above theorem is also valid if one replaces $X^{+}$, wherever it occurs, by $X^{-}$. To verify this it is sufficient to simply apply the above theorem to the problem obtained after the change of variables $s=-x$.

We now show that the LP criteria of Weyl [6], Friedrichs [3] and Sears [4] follow from the above by proper selection of the comparison operator.

For the first two LP criteria let $\hat{q}=0$. The solutions of $\hat{L} v=0$ are then linear combinations of $v_{1}=1$ and $v_{2}=\int_{x 0}^{x}(p(s))^{-1} d s$. Clearly $\hat{L}$ is nonoscillatory. Furthermore, $\hat{L}$ will be $\mathrm{LP}$ at $X^{+}$if either $X^{+}=\infty$ (since then $v_{1}=1$ is not in $L^{2}$ ) or if $X^{+}<\infty$ and $v_{2}$ is not in $L^{2}$. Thus we have rederived the following:

Corollary 1 (Weyl [7, p. 27]). L is LP at $X^{+}=\infty$ if $q$ is bounded below at $X^{+}=\infty$.

Corollary 2 (Friedrichs [3, p. 13]). L is LP at $X^{+}<\infty$ if $q$ is bounded below at $X^{+}$and $\int_{x_{0}}^{x}(p(s))^{-1} d s$ is not in $L^{2}\left(x_{0}, X^{+}\right)$.

For the Sears LP criterion let $p=1$ and $\hat{q}=c / x^{2}$ where $c$ is a constant as yet unspecified. The solutions of $\hat{L} v=0$ are then linear combinations of $v_{1}=x^{\gamma_{1}}$ and $v_{2}=x^{\gamma_{2}}$ where $\gamma_{1,2}=1 / 2 \pm(c+1 / 4)^{1 / 2}$. Clearly $\hat{L}$ is nonoscillatory if $c>-1 / 4$. If $c \geqq 3 / 4$ then $v_{2}$ is not in $L^{2}\left(0, x_{0}\right)$ so that $\hat{L}$ is then LP at $X^{-}=0$. We have thus rederived

Corollary 3 (Sears [4]). L defined by

$$
L y=-y^{\prime \prime}+q y
$$

is LP at $X^{-}=0$ if $q-3 / 4 x^{2}$ is bounded below at $X^{-}=0$.

It is interesting to note that the above theorem can be derived from the LP criterion of Weyl in Corollary 1 when use is made of the following Liouville-type transformation:

Lemma. (See, for example, [3, p. 16].)

$$
L y=-\left(p y^{\prime}\right)^{\prime}+q y, \quad x_{0}<x<X^{+},
$$

becomes 


$$
\tilde{L} u=(-d / d t)(\tilde{p}(d u / d t))+\tilde{q} u, \quad 0<t<T^{+},
$$

by the transformation

$$
y=v u, \quad t(x)=\int_{x_{0}}^{x} v^{2}(s) d s
$$

with $v$ as yet unspecified where

$$
\tilde{p}=v^{4} p, \quad \tilde{q}=q-\frac{\left(p v^{\prime}\right)^{\prime}}{v}, \quad T^{+}=\int_{x_{n}}^{x^{+}} v^{2}(s) d s .
$$

Furthermore,

$$
\int_{x_{0}}^{x^{+}} y^{2}(x) d x=\int_{0}^{T^{+}} u^{2}(t) d t .
$$

Thus $\tilde{L}$ is LP at $T^{+}$if and only if $L$ is LP at $X^{+}$.

Now let $v$ in the above Lemma be a solution of the comparison equation

$$
\hat{L} v=-\left(p v^{\prime}\right)^{\prime}+\hat{q} v=0 .
$$

Then clearly $\tilde{q}=q-\hat{q}$.

If $\hat{L}$ is $\mathrm{LP}$ at $X^{+}$then one can select $v$ so that $T^{+}=\infty$.

If $\hat{L}$ is nonoscillatory at $X^{+}$then $\tilde{p}=v^{4} p>0$.

Thus the hypothesis of the theorem insures that one can apply the LP criterion of Weyl to conclude that $\tilde{L}$ is LP at $T^{+}=\infty$. Hence by the above Lemma $L$ is LP at $X^{+}$. Q.E.D.

One can generalize Corollaries 2 and 3 by introducing a new independent variable

$$
t(x)=\int^{x} d s / p(s)
$$

and setting

$$
\hat{q}(x)=h(t) / p(x),
$$

where the function $h(t)$ is as yet unspecified. Then

$$
p(x) \hat{L} v=-d^{2} v / d t^{2}+h(t) v .
$$

Each $h(t)$ for which $\hat{L}$ is nonoscillatory and LP at $X^{+}$or $X^{-}$then leads, via the above theorem, to an LP criterion for $L$. For example, if one selects

$$
h(t)=C / t^{2}
$$


then $\hat{L} v=0$ has solutions $t^{\gamma_{1}}$ and $t^{\gamma_{2}}$ where $\gamma_{1}$ and $\gamma_{2}$ are the values indicated in the above discussion of Corollary 3. One thus concludes the following:

Corollary 4. L defined in (1) is LP at $X^{+}\left(X^{-}\right)$if $C \geqq 1 / 4$ is such that (i) at least one of $t^{\gamma_{1}}$ and $t^{\gamma_{2}}$ is not in $L^{2}\left(x_{0}, X^{+}\right)\left(L^{2}\left(x_{0}, X^{-}\right)\right)$and (ii) $q(x)-C / p(x) t^{2}$ is bounded below at $X^{+}\left(X^{-}\right)$where $t$ is defined in (2).

Corollary 2 corresponds to the selection $C=0$. Then $\gamma_{1}=1$ and $\gamma_{2}=0$ so that condition (i) becomes simply that $t$ is not in $L^{2}$.

Corollary 3 corresponds to the selection $p(x)=1$. This results in $t(x)=x$ and the optimum value of $C$ is seen to be $3 / 4$.

As a further example of Corollary 4 let $p(x)=x^{\alpha}$ where $\alpha$ is a real constant. (Corollary 2 will not be applicable to this case if $\alpha<3 / 2$ since then $t(x)$ will be in $L^{2}$.) From (2), (3) and (5)

$$
\hat{q}(x)=K / x^{2-\alpha}
$$

where $K=(1-\alpha)^{2} C$. It is then easily seen that $\hat{L}$ is nonoscillatory and LP at $X^{-}=0$ if $K \geqq(3-2 \alpha) / 4$. This then proves

Corollary 5. L defined by (1) is LP at $X^{-}=0$ if $p=x^{\alpha}, \alpha<3 / 2$ and $q-(3-2 \alpha) / 4 x^{2-\alpha}$ is bounded below at $X^{-}=0$.

Corollary 3 corresponds to the special case $\alpha=0$.

In order to facilitate comparison with Corollary 2 one can rewrite Corollary 5 by replacing $x$ by $X^{+}-x$ and $X^{-}=0$ by $X^{+}<\infty$.

A selection of $h(t)$ in (3) more general than (5), and yet which is readily dealt with, is one for which $\hat{L} v=0$ has a regular singularity at $T$ where $T=\lim t(x)$ as $x \rightarrow X^{-}$. Thus if $T=0$ let

$$
h(t)=t^{-2}\left(C+C_{1} t+\cdots+C_{k} t^{k}\right)
$$

while if $T=\infty$ let

$$
h(t)=t^{-2}\left(C+C_{1} t^{-1}+\cdots+C_{k} t^{-k}\right) .
$$

In either case, as long as $\gamma_{1}-\gamma_{2}$ is not an integer, $t^{\gamma_{1}}$ and $t^{\gamma_{2}}$ are now the leading terms of two solutions of $\hat{L} v=0$. Hence in Corollary 4 one can replace (ii) by (ii) $q(x)-h(t) / p(x)$ is bounded below at $X^{+}\left(X^{-}\right)$ where $C$ is described in Corollary 4 but $C_{1}, C_{2}, \cdots, C_{k}$ are unrestricted.

\section{REFERENCES}

1. J. C. Burkhill, The theory of ordinary differential equations, Oliver and Boyd, London, 1962; p. 26.

2. E. A. Coddington and N. Levinson, Theory of ordinary differential equations, McGraw-Hill, New York, 1955; p. 225. 
3. K. O. Friedrichs, Über die ausgezeichnete Randbedingung in der Spektraltheorie der halbbeschränkten gewöhnlichen Differentialoperatoren zweiter Ordnung, Math. Ann. 112 (1935), 1-23.

4. D. B. Sears, On the solutions of a second order differential equation which are integrable square, J. London Math. Soc. 24 (1949), 207-215.

5. F. G. Tricomi, Differential equations, Hafner, New York, 1961; p. 103.

6. H. Weyl, Über gewöhnliche Differentialgleichungen mit Singularitäten und die zugehörigen Entwicklungen willkürlicher Funktionen, Math. Ann. 68 (1910), 220-269. Reprinted in Selecta Hermann Weyl, pp. 9-58, Birkhauser, Basel, 1952.

7. - Über das Pick-Nevalinnasche Interpolationsproblem und sein infinitesmales Analogen, Ann. Math. 36 (1935), 230-254. Reprinted in Selecta Hermann Weyl, pp. 405-430, Birkhauser, Basel, 1952.

ADELPHI UNIVERSITY 